\title{
Detection and Predictors of Atrial Fibrillations in patients after pacemaker implantations.
}

\author{
Michal Chudzik ${ }^{1, \mathrm{~A}-\mathrm{B}}$, Anna Nowek ${ }^{1, \mathrm{~B}}$ \\ 1 Department of Electrocardiology, Medical University of Lodz, Poland
}

Address for correspondence:

Michal Chudzik, Department of Electrocardiology, Medical University of Lodz, Poland email: michalchudzik@wp.pl

Anna Nowek Department of Electrocardiology, Medical University of Lodz, Poland email: noweka@wp.pl

Received: 21.12 .2016

Revised: 26.12.2016

Accepted: 27.12.2016

\section{Key words:}

\section{AliveCor ECG; electrocardiogram; 12-lead; smart phone ECG}

\section{Introduction}

Atrial fibrillation (AF) is the most common sustained cardiac rhythm disturbance in adults and the leading cardiac cause of morbidity and mortality ${ }^{[1]}$. Dual-chamber pacemakers (PM) have the ability to document even brief episodes of $\mathrm{AF}^{[2]}$.

Assessment of the prevalence of silent paroxysmal AF is still a challenge, especially in patients without symptoms ${ }^{[3,4]}$.

The presence of AF increases the risk of stroke and embolism. One-quarter of ischaemic strokes are of unknown cause, usually designated cryptogenic, and undiagnosed AF may be a potential aetiological factor. While AF typically presents with palpitations, dyspnoea, chest pain, and fatigue, it may also be asymptomatic ${ }^{[5,6]}$

Most of these episodes are short and asymptomatic and would not be detected by ways other than a pacemaker ${ }^{[7]}$. The detection of undiagnosed atrial tachycardia/atrial fibrillation (AF) could be useful for primary prevention of consequences and complications such as stroke, by including early treatment and intervention. The application of anticoagulation therapy in asymptomatic patients with device-detected AF and the CHA2DS2VASc score should be recommended and could prevent stroke.

AF episodes with implanted devices lasting $\geq 5$ minutes have been proven to be an independent predictor of death or nonfatal stroke in these patients ${ }^{[7]}$.

All available data supporting the use of oral anticoagulation for stroke prevention in AF have been obtained from patients with clinically diagnosed (ECG documented) $\mathrm{AF}^{[8,9,10]}$. It is still unknown how many patients with PM-detected AF have indications for anticoagulation therapy.
The aim of this study was to document the prevalence of pacemaker-detected AF in patients without atrial arrhythmias before implantations and to estimate the CHA2DS2VASc score to start anticoagulation therapy.

We sought to document frequency of AF, time to new diagnosis dtetermined by pacemaker data storage as well as clinical and echocardiographic risk predictors for development of pacemaker-detected AF.

\section{Methods}

Patients after de novo implantation of dual-chamber PM capable of documenting atrial tachycardia (AT) without a history of paroxysmal atrial tachyarrhythmia were included in this study. Manufacturer-specific nominal settings for AT detection were typically employed. AT episodes were defined as atrial burden episodes (AFburden). Exclusion criteria were the following: replacement devices, any documented AT episode before implantation, unable or unwilling to consent. Patients were followed up at 3.6 and 12 months after implantation. Diagnostic information with AT/AF burden measurements was collected and presence of symptoms was noted at every follow-up visit. In every patient confirmation of AT with intracardiac electrocardiogram (IEGM) was programmed to record the maximum number of recorded episodes of high frequency atrial episodes. Episodes of AT were classified as atrial fibrillation only if all recorded episodes in IEGM were evaluated and confirmed by the physician as AF.

The following data were analysed: age, gender, BMI, NYHA class, indication for implantation (divided into three groups: bradycardia-tachycardia syndrome, sick sinus syn- 
drome (SSS), atrioventricular block (AVB)), coexisting diseases such as coronary artery disease, past myocardial infarction, diabetes, hypertension, stroke, and pharmacotherapy. Echocardiograms were performed before implantation and 1 year after, and they were interpreted by a single reader, blinded to the presence of pacemaker-detected AT. The following echocardiographic measures were evaluated: left and right atrium dimensions, ejection fraction, left ventricular systolic diameter, left ventricular diastolic diameter, right ventricular diastolic diameter, mitral regurgitation, and tricuspid regurgitation. Regarding data retrieved from the pacemaker memory, AF burden $\%$, atrial pacing $\%(\mathrm{AP} \%)$ and ventricular pacing $\%$ $(\mathrm{VP} \%)$ were documented at every visit.

Normality of the data set was tested using the Shapiro-Wilk test. Quantifiable variables were expressed as mean \pm standard deviation (SD) or median and inter-quartile range (IQR) depending on the results of the normality test. Differences between two independent samples for continuous clinical data were analysed using Student's t-test and the Mann-Whitney $\mathrm{U}$ test. For categorical variables statistical analysis was based on the chi-squared test and chi-squared test with Yates' adjustment. Risk predictors with a $\mathrm{p}$ value $<0.05$ in univariate analyses were included in the multivariate logistic regression model. The results were considered significant for $\mathrm{p}<0.05$. STATISTICA 10 and SPSS 21 software packages were used to analyse the data.

\section{Results}

\section{Prevalence and frequency of AF:}

In the study there participated 61 patients without clinical history of AF. In 24 out of 61 (39\%) patients the indication for implantation was SSS and in 37 (61\%) it was AV block. During the 12-month follow-up AF was detected by the pacemaker in 11 of $61(18 \%)$ patients - in 5 patients with SSS and in 6 with AV block. So in $18 \%$ pacemaker detection of AF was the first paroxysmal atrial tachyarrhythmia diagnosis.

No persistent or permanent $\mathrm{AF}$ was diagnosed during the 12-month follow-up.

\section{Time to detection of AF:}

The average time to detection of AF episodes was $2.3 \pm 4.3$ months from the beginning of the follow-up. The majority of AF episodes were detected after 3 months - in 5 (45\%) out of 11 patients. Next 4 cases (36\%) of AF burden were detected at 6 months and 2 patients at the 12-month follow-up.

Symptoms such as palpitations and dyspnoea at any of the follow-ups were reported in 6 (54\%) out of 11 cases.

Symptoms were reported by significantly more women than men ( $89 \%$ vs $11 \%, p=0.005)$. Asymptomatic episodes (pacemaker-detected AF without symptoms reported from the patient during any of the follow-ups) occurred in 5 patients ( 2 men and 3 women).

\section{Risk factors of AF:}

Multivariate analysis showed that clinical factors such as age, BMI, NYHA class, coexisting diseases such as coronary artery disease (CAD), past myocardial infarction (MI), diabetes (DM), hypertension (HA) and stroke, as well as pharmacotherapy, were statistically nonsignificant as risk factors for AF. The comparison of clinical data is shown in Table 1.

\begin{tabular}{|c|c|c|c|}
\hline Variable & $\begin{array}{l}\text { No AF } \\
\text { detected } \\
(n=50)\end{array}$ & $\begin{array}{l}\text { Pacemaker } \\
\text { AF } \\
\text { detected } \\
\text { (n=11) }\end{array}$ & p value \\
\hline Age (years) & 74.65 & 73.63 & 0.432 \\
\hline BMI & 27.67 & 28.72 & 0.435 \\
\hline CAD & 21 & 5 & 0.413 \\
\hline MI & 5 & 2 & 0.658 \\
\hline DM & 10 & 5 & 0.343 \\
\hline HA & 39 & 10 & 0.654 \\
\hline ACEI & 42 & 7 & 0.898 \\
\hline statins & 16 & 5 & 0.960 \\
\hline anti-arrhythmic & 0 & 0 & 0.213 \\
\hline VKA & 2 & 0 & 0.976 \\
\hline beta-blocker & 36 & 7 & 0.087 \\
\hline LA (mm) & $57 / 46$ & $59 / 49$ & 0.549 \\
\hline $\mathrm{RA}(\mathrm{mm})$ & $51 / 38$ & $55 / 40$ & 0.512 \\
\hline $\mathrm{EF} \%$ & 64.60 & 62.38 & 0.194 \\
\hline LVSD (mm) & 31.6 & 30.0 & 0.437 \\
\hline LVdD (mm) & 50.8 & 50.6 & 0.964 \\
\hline $\mathrm{RVdD}(\mathrm{mm})$ & 26.0 & 25.8 & 0.516 \\
\hline $\mathrm{AP} \%$ & 51.2 & 26.1 & 0.321 \\
\hline VP\% & 54.2 & 61.5 & 0.268 \\
\hline \multicolumn{4}{|c|}{$\begin{array}{l}\text { BMI - body mass index, CAD - coronary artery disease, MI - myocardial infarction, } \\
\text { DM - diabetes mellitus, HA - arterial hypertension, ACEI - angiotensin-converting- } \\
\text { enzyme inhibitor, VKA - vitamin K antagonists, LA - left atrium dimensions prior to } \\
\text { implantation, RA - right atrium dimensions prior to implantation, EF - ejection } \\
\text { fraction, LVsD - left ventricular systolic diameter, LVdD - left ventricular diastolic } \\
\text { diameter, RVdD - right ventricular diastolic diameter, AP\% - atrial pacing, VP\% - } \\
\text { ventricular pacing }\end{array}$} \\
\hline
\end{tabular}

There was no significant difference in echocardiographic parameters (left and right atrial dimensions (LA, RA), ejection fraction $(\mathrm{EF})$, left ventricular systolic diameter (LVsD), left ventricular diastolic diameter (LVdD), right ventricular diastolic diameter (RVdD), mitral regurgitation (IM), tricuspid regurgitation (IT)) between the group with pacemaker-detected AF and the group without pacemaker-detected AT. ECHO parameters are shown in Table 1. Data retrieved from 
pacemaker memory - atrial pacing $\%(\mathrm{AP} \%)$ and ventricular pacing \% (VP\%) - were statistically nonsignificant.

\section{CHA2DS2-VASc risk score:}

In every patient with AF detected the CHA2DS2-VASc score was $\geq 3$. It was 3 in 5 patients, 4 in 2 patients, 5 in 1 patient, 6 in 2 patients and 7 in 1 patient.

The CHA2DS2-VASc risk score for susceptibility to the development of thrombo-embolic complications in all patients with $\mathrm{AF}$ was $\geq 1$, which means oral anticoagulant therapy was indicated in all these patients, since the diagnosis of AF was made.

Oral anticoagulant OAC therapy was given to 8 patients (77\%) who experienced AF. In the majority of patients (in 4 patients) OAC was introduced at 3 months of follow-up, in 3 patients at 6 months of follow-up, and in 1 patient at 12 months of follow-up. During the follow-up period none of the patients developed any thrombo-embolic complications.

\section{Discussion}

The main finding of our study was that newly detected AF during the first year after implantation was documented in more than $18 \%$ of patients. In all of these patients the CHA2DS2-VASc risk score was $\geq 3$ with the indication for OAC, but OAC was given only to 8 out of 11 patients. In 2 patients because of very high risk of haemorrhage, with a history of serious bleeding, OAC were not started. One patient refused. Possibly due to detection of asymptomatic AF episodes and early treatment of OAC during the follow-up period none of the patients developed any thrombo-embolic complications. In terms of symptoms, analysis of data stored by the pacemaker showed that AF episodes often do not correlate with complaints.

Pacemaker diagnostic data with intraatrial EGMs can diagnose specific atrial tachyarrhythmias and identify other pacemaker-sensed events ${ }^{[11]}$. The diagnostic accuracy of pacemaker and ICDs with an atrial lead is very high, with appropriate detection of $95 \%$ of AF episodes, while for implantable loop recorders the specificity is about $85 \%{ }^{[12]}$. Our findings correlate with other studies with higher numbers of patients included. Ziegler et al..$^{[13]}$ documented that continuous PM-based monitoring identified newly detected AF in $30 \%$ of patients with stroke risk factors and episodes occurred sporadically, highlighting the difficulty in detecting paroxysmal AF using traditional monitoring methods such as ECG or 24-hour Holter ECG monitoring ${ }^{[10]}$. In the Yedlapati et al. study which included 728 patients with DDD pacemakers, 26\% out of 66 patients with AF recorded were newly discovered to have AF, which was similar to our finding ${ }^{[14]}$.

The importance of first-year observation was also noted by Nagarakanti et al. The time to the first, second, and third persistent AT recurrences progressively decreases with a high likelihood of established persistent AT within 9 months of onse ${ }^{[15]}$. It was also documented that AF burden increases progressively over the long term ${ }^{[15]}$.
Patients with AF are more likely to have adverse clinical outcomes, including a higher incidence of stroke, death, and subsequent $\mathrm{AF}$ than patients without $\mathrm{AF}^{[1]}$. Analysis showed that in a relatively unselected population of patients with arrhythmia detecting devices, daily AF burden is associated with an increased risk of ischaemic stroke or TIA even after adjustment for use of oral anticoagulants ${ }^{[16]}$. These data add to current evidence that measuring daily AF burden may have important clinical relevance and support the search for specific thresholds of AF burden associated with a substantial increase in the risk of stroke ${ }^{[17,18]}$. Integration of AF presence/duration/ burden has the potential to contribute to improved clinical risk stratification, and its aid to clinical decision-making should be tested prospectively ${ }^{[19,20]}$. About $25-30 \%$ of patients presenting with strokes have AF that was not previously recognized ${ }^{[21]}$. Furthermore, AF has been reported to be associated with substantial deterioration in attention and memory, even in the absence of a history of stroke and obvious defects on computer tomograms. The stroke risk conferred by paroxysmal AF has not been well characterized but has been claimed to be the same as continuous AF. This establishes the need for better detection of atrial arrhythmias, and device-based monitoring gives us the possibility for continuous rhythm control compared to traditional ECG or 24-hour Holter ECG monitoring. Unfortunately the short-term follow-up in our study resulted in absence of clinical events (e.g. stroke or death), so we could not assess the risk of adverse events. Data from the TRENDS study do not allow us to define a "safe" AF burden threshold that confers a risk no greater than that of zero AF burden ${ }^{[6]}$. In this study there was also no threshold for AT/AF burden episodes.

One of the study findings is that there are no clinical predictors of AF. Healey et al. found no, other than older age, clinical predictors of pacemaker-detected AT in a group of 445 patients with no previous history of $\mathrm{AF}$ and a high percentage $(50 \%)$ of device-detected $\mathrm{AT}^{[2]}$.

Among patients with symptomatic bradycardia and a history of atrial fibrillation, symptoms of atrial fibrillation often were not associated with documented atrial tachyarrhythmias (positive predictive value $17 \%$ in the Strickberger SA study), and more than $90 \%$ of atrial tachyarrhythmias were clinically silent (AT/AF episodes without symptoms such as palpitations or dyspnoea reported by the patient) ${ }^{[17]}$. In our study asymptomatic episodes occurred in 5 patients (44\%). Due to the symptoms reported by patients without a recorded atrial burden, the association of episodes and symptoms is uncertain.

\section{Study Limitations}

The results of this single-centre study may not be directly applicable to other practice settings. The study was limited to patients with pacemakers with normal ejection fraction. Atrial undersensing can occur during AT/AF episodes, which 
can lead to failure to detect AT/AF burden episodes. Based on pacemaker diagnostics and short IEGM recording it can also be difficult to differentiate AF from other forms of atrial arrhythmias. Additionally, it would be very beneficial to use telemedicine to detect AF episodes as soon as possible. Remote monitoring provides a very short reaction time to $\mathrm{AF}$ alerts ${ }^{[22.23]}$. Finally, our study is limited by the short-term follow-up resulting in absence of clinical events (e.g. stroke or death).

\section{Conclusions}

The diagnostic accuracy of a pacemaker with an atrial lead is very high and pacemaker detection of $\mathrm{AT}$ is often the first paroxysmal atrial tachyarrhythmia diagnosis. Newly detected $\mathrm{AT} / \mathrm{AF}$ during first year after implantation was documented in more than $15 \%$ of patients, and no clinical characteristics in patients without a history of AF prior to implantation were associated with pacemaker-detected AF. In all patients with AT according to the CHA2DS2-VASc score indications for anticoagulation therapy were found.

Frequent follow-ups during the first year after implantation are very important for new diagnosis of arrhythmias thanks to the possibilities of patients' continuous monitoring by means of implantable cardiac devices.

\section{References}

1. Glotzer TV, Hellkamp AS, Zimmerman J, et al. Atrial high rate episodes detected by pacemaker diagnostics predict death and stroke: report of the atrial diagnostics ancillary study of the mode selection trial (MOST). Circulation 2003; 107:1614-1619.

2. Healey JS, Martin JL, Duncan A, et al. Pacemaker-detected atrial fibrillation in patients with pacemakers: prevalence, predictors and current use of oral anticoagulation. Can J Cardiol 2013; 29:224-228.

3. Savelieva I, Camm AJ. Clinical relevance of silent atrial fibrillation: prevalence, prognosis, quality of life, and management. J Intervent Card Electrophysiol 2000; 4:369-82.

4. Page RL, Wilkinson WE, Clair WK, McCarthy EA, Pritchett EL. Asymptomatic arrhythmias in patients with symptomatic paroxysmal atrial fibrillation and paroxysmal supraventricular tachycardia. Circulation 1994;89: 224-7.

5. Healey JS, Conolly SJ, Gold MR, et al. Pacemaker-detected atrial tachyarrhythmias and the risk of stroke. N Engl J Med 2012; 366:122-131.

6. Glotzer TV, Daound EG, Wyse DG, et al. The relationship between daily atrial tachyarrhythmia burden from implantable device diagnostics and stroke risk: the TRENDS study. Circ Arrhythm Electrophysiol 2009; 2:474-80.

7. Capucci A, Santini M, Padeletti L, et al. Monitored atrial fibrillation duration predicts arterial embolism events in patients suffering from bradycardia and atrial fibrillation implanted with antitachycardia pacemakers. J Am Coll Cardiol. 2005;46:1913-1920

8. Ruff CT, Giugliano RP, Braunwald E, Hoffman EB, Deenadayalu N, Ezekowitz MD et al. Comparison of the efficacy and safety of new oral anticoagulants with warfarin in patients with atrial fibrillation: a meta-analysis of randomised trials. Lancet 2014; 383:955-62.

9. Granger $\mathrm{CB}$, Alexander JH, McMurray JJ, Lopes RD, Hylek EM, Hanna $M$ et al. Apixaban versus warfarin in patients with atrial fibrillation. N Engl J Med 2011;365:981-92.

10. Patel MR, Mahaffey KW, Garg J, Pan G, Singer DE, Hacke W etal. Rivaroxaban versus warfarin in nonvalvular atrial fibrillation. N Engl J Med 2011;365:883-91.

11. Pollak WM, Simmons JD, Interian A Jr, et al. Clinical utility of intraatrial pacemaker stored electrograms to diagnose atrial fibrillation and flutter. Pacing Clin Electrophysiol 2001; 24(4 Pt 1):424-429.

12. Boriani G, Glotzer TV, Santini M, et al. Device-detected atrial fibrillation and risk for stroke: an analysis of $>10$ 000 patients from the SOS AF project (Stroke prevention strategies based on atrial fibrillation information from implanted devices). Eur Heart J 2014; 35:508-516.

13. Ziegler PD, Glotzer TV, Daoud EG, et al. Detection of previously undiagnosed atrial fibrillation in patients with stroke risk factors and usefulness of continuous monitoring in primary stroke prevention. Am J Cardiol 2012; 110(9):1309-14.

14. Yedlapati N, Fisher JD. Pacemaker diagnostics in atrial fibrillation: limited usefulness for therapy initiation in a pacemaker practice. Pacing Clin Electrophysiol 2014 Apr 12, doi:10.1111/pace.12392.

15. Nagarakanti R, Saksena S, Hettrick D, Koehler JL, Grammatico A, Padeletti L. Progression of new onset to established persistent atrial fibrillation: an implantable device-based analysis with implications for clinical classification of persistent atrial fibrillation. J Interv Card Electrophysiol 2011; 32(1):7-15.

16. Gillis AM, Morck M. Atrial fibrillation after DDDR pacemaker implantation. J Cardiovasc Eltectrophysiol 2002; 13(6):542-547.

17. Strickberger SA, Ip J, Saksena S, Curry K, Bahnson TD, Ziegler PD. Relationship between atrial tachyarrhythmias and symptoms. Heart Rhythm 2005; 2(2): 125-131.

18. Healey JS, Conolly SJ, Gold MR, et al. Subclinical atrial fibrillation and the risk of stroke. N Engl J Med 2012; 366:120-129.

19. Lamas G. How much atrial fibrillation is too much atrial fibrillation? N Engl J Med 2012; 366:178-180.

20. Boriani G, Botto GL, Padeletti L, et al. Improving stroke risk stratification using the CHADS2 and CHA2DS2VASc risk scores in patients with paroxysmal atrial fibrillation by continuous arrhythmia burden monitoring. Stroke 2011; 42(6):1768-70.

21. Benezet-Mazuecos J, Rubio E, Farre J. Atrial High rate episodes in patients with dual-chamber cardiac 
implantable electronic devices: unmasking silent atrial fibrillation. Pace 2014; 37(8):1080-1086.

22. Shacham J1, Birati EY, Malov N, et al. Telemedicine for diagnosing and managing paroxysmal atrial fibrillation in outpatients. The phone in the pocket. Int J Cardiol 2012; 157(1):91-95.

23. Ricci RP. Disease management: atrial fibrillation and home monitoring. Europace. 2013; 15 Suppl 1:i35-i39. 